\title{
PROBABILISTIC INTERPRETATION OF COMPLEX FUZZY SET
}

\author{
Nilambar Sethi ${ }^{1}$, S. K. Das ${ }^{2}$ and D.C. Panda ${ }^{3}$ \\ ${ }^{1}$ Department of Information Technology \\ Gandhi Institute of Engineering \& Technology, Gunupur, Orissa \\ nilambar_sethi@rediffmail.com \\ ${ }^{2}$ Department of Computer Science, Berhampur University, Berhampur, Orissa \\ dr.dassusanta@yahoo.co.in \\ ${ }^{3}$ Department of Electronics \& Communication Engineering \\ Jagannath Institute of Technology \& Management, Paralakhemundi, Orissa \\ d_c_panda@yahoo.com
}

\begin{abstract}
The innovative concept of Complex Fuzzy Set is introduced. The objective of the this paper to investigate the concept of Complex Fuzzy set in constraint to a traditional Fuzzy set, where the membership function ranges from [0,1], but in the Complex fuzzy set extended to a unit circle in a complex plane, where the member ship function in the form of complex number. The Compressive study of mathematical operation of Complex Fuzzy set is presented. The basic operation like addition, subtraction, multiplication and division are described here. The Novel idea of this paper to measure the similarity between two fuzzy relations by evaluating $\delta$-equality. Here also we introduce the probabilistic interpretation of the complex fuzzy set where we attempted to clarify the distinction between Fuzzy logic and probability.
\end{abstract}

\section{KEY WORD}

Complex fuzzy set, Complex fuzzy relation, complement of complex fuzzy relation, $\delta$-equality, probability.

\section{INTRODUCTION}

The concept of the fuzzy set was first introduced by Zadeh in a seminal paper in 1965 [7]. This is the generalization of script Set in terms of membership function. The notion of the fuzzy set A on the universe of discourse $\mathrm{U}$ is the set of order pair $\left\{\left(\mathrm{x}, \mu_{\mathrm{A}}(\mathrm{x})\right), \mathrm{x} \varepsilon \mathrm{U}\right\}$ with a membership function $\mu_{\mathrm{A}}(\mathrm{x})$, taking the value on the interval [0,1]. Ramot et al [5], extended the fuzzy set to complex fuzzy set with membership function, $z=r_{s} e^{i w_{s}(x)}$ where $i=\sqrt{-1}$, which ranges in the interval [0, 1] to a unit circle. Ramot et al [4], also introduced different fuzzy complex operations and relations, like union, intersection, complement etc., Still it is necessary to determine the membership functions correctly, which will give the appropriate or approximate result for real life applications. The membership function defined for the complex fuzzy set $z=r_{s} e^{i w_{s}(x)}$, which compromise an amplitude term $\mathrm{r}_{\mathrm{s}}(\mathrm{x})$ and phase term $\mathrm{w}_{\mathrm{s}}$. The amplitude term retains the idea of "fuzziness" and phase term signifies declaration of complex fuzzy set, for which the second dimension of membership is required. The complex fuzzy set allows extension of fuzzy logic that 
is $i$ to continue with one dimension gradeness of membership. Xin Fu et al [8] defined the fuzzy complex membership function of the form $\mathrm{z}=\mathrm{a}+\mathrm{ib}$, where $\mathrm{x}, \mathrm{y}$ are two fuzzy numbers with membership function $\mu_{\mathrm{A}}(\mathrm{x}), \mu_{\mathrm{B}}(\mathrm{x})$ respectively. If $\mathrm{b}$ does not exist, $\mathrm{z}$ degenerates to a fuzzy number.

$\mathrm{Xin} \mathrm{Fu}$ et al [8], also discussed a complex number in cartesian form where $\mathrm{a}=\mathrm{r}_{\mathrm{s}} \cos (\mathrm{x})$ and $\mathrm{b}=\mathrm{r}_{\mathrm{s}} \sin (\mathrm{x})$, which are in polar forms defined in [4]. The fuzzy number is created by interpolating complex number in the support of fuzzy set ([1], [2], [3][6]).The member ship function are usually difficult to determine accurately and one may argue of accurate or precise membership function are necessary in reality. In order to characterized in accurate membership function in more general Cai(1995) proposed and discussed $\delta$-equality of the fuzzy set.

Following the above resent development the fuzzy set theory Zhang et al (2004) studies $\delta$ equality of complex fuzzy set. This is the logical development since a complex membership function should more difficult that a real membership function to be determine in practice.

Complex fuzzy set is a unique framework over the advantage of traditional fuzzy set .The support of complex fuzzy set is unrestricted, may include any kind of object such as number, name etc, which is off course a complex number. The notion of T-norm and T-conorm are used through out this paper [4].

This paper is organized in the following order. The review of complex fuzzy set and operations on it are discussed in section 2 and section 3 the $\Delta$-Equalities of complex Fuzzy Relations A novel idea to probabilistic interpretation are studied in section 4 followed by summary and suggestion for future work are given in section 5 .

\section{COMPLEX FUZZY SET AND RELATION}

\subsection{Concept \& Operations}

Complex fuzzy set is the basis for complex fuzzy logic [7]. The formal definition of complex fuzzy set was provided in [7] followed by a discussion and interpretation of the moral concept. In addition, several set theoretic operations on complex fuzzy set were discussed with several examples, which illustrate the potential applications of complex fuzzy set in information processing.

\subsection{Definition of Complex Fuzzy Set}

A complex fuzzy set $\mathrm{S}$, defined on a universe of discourse $\mathrm{U}$, is characterized by a membership function $\mu_{s}(x)$, which can assign any element $\mathrm{x} \in \mathrm{U}$ as a complex valued grade of membership in $\mathrm{S}$. The value of $\mu_{s}(x)$ lies in a unit circle in the complex plane and in the form $z=r_{s} e^{i w_{s}(x)}$, where $i=\sqrt{-1}$ and $\mathrm{r}_{\mathrm{s}} \in[0,1]$.

The complex fuzzy set S, may be defined as the set of order pair given by

$$
S=\left\{\left(x, \mu_{s}(x)\right): x \in U\right\}
$$




\subsection{Complex Fuzzy Union}

\subsubsection{Definition $([6,7])$}

In the traditional fuzzy logic, union for two fuzzy set $A$ and $B$ on $U$ denoted as $A \cup B$ is specified by a function $\mu$, where $\mu:[0,1] \times[0,1] \rightarrow[0,1]$.

The membership function $\mu_{(\mathrm{A} \cup \mathrm{B})}$, may be defined as one of followings.

$$
\begin{array}{ll}
\text { (i) Standard Union } & \mu_{A U B}(x)=\max \left[\mu_{A}(x), \mu_{B}(x)\right] \\
\text { (ii) Algebraic Sum } & \mu_{A U B}(x)=\mu_{A}(x)+\mu_{B}(x)-\mu_{A}(x) \cdot \mu_{B}(x) \\
\text { (iii) Bound Sum } & \mu_{A U B}(x)=\min \left[1, \mu_{A}(x)+\mu_{B}(x)\right]
\end{array}
$$

The fuzzy union must satisfy the definition 2.2 and is equivalent to the proposed T-Co norms [9].

\subsubsection{Properties}

The Complex fuzzy union is defined by a set of axioms. These axioms represent properties of complex fuzzy union functions that must satisfy in order to intuitively acceptable values.

\section{(a) Complex fuzzy union does not satisfy the closure property.}

In the algebraic sum union function does not satisfy the closure property for complex fuzzy set, which can be realized from the following example.

Example 2.3.1. $\mu_{A}(x)=\mu_{B}(x)=i$

$$
\Rightarrow \mu_{A \cup B}{ }^{(x)}=\mu_{A}{ }^{(x)}+\mu_{B}{ }^{(x)}-\mu_{A}{ }^{(x)} \cdot \mu_{B}{ }^{(x)}=1+2 i
$$

It shows that the membership function lies outside of the unit circle. i.e. $\sqrt{1^{2}+2^{2}}=\sqrt{5}>1$.

\section{(b) Complex Fuzzy union must be monotonic}

Since complex number is not linearly order, monotonicity of the complex numbers is required.

Example 2.3.2. Suppose $b \leq d \Rightarrow \mu(a, b) \leq \mu(a, d)$ using definition2.3.1 (2)

Similarly the max and min operators used in (3) \& (5) are not applicable to complex valued grades of membership. Here we can apply traditional fuzzy definition of union by keeping same approach to complex part and different approach to phase may be defined in the following way.

\subsubsection{Definition $([6,7])$}

Suppose A and B be two complex fuzzy set on $U$ with complex valued membership function $\mu_{n}(x), \mu_{B}(x)$ respectively. Then complex fuzzy union $\mathrm{A} \cup \mathrm{B}$ may be defined as

$$
\mu_{(A \cup B)}(x)=\left[r_{A}(x) \oplus r_{B}(x)\right] e^{i w_{A \cup B}(x)}
$$

where $\oplus$ represents the T-Conorm and $W_{A \cup B}$ is defined as follows. 
a) (Sum) $W_{A \cup B}=W_{A}+W_{B}$

b) $\quad(\operatorname{Max}) W_{A \cup B}=\max \left(W_{A}, W_{B}\right)$

c) (Min) $W_{A \cup B}=\min \left(W_{A}-W_{B}\right)$

and

d) (Winner takes all) $W_{A U B}=\left\{\begin{array}{lll}W_{A} & \text { if } r_{A}>r_{B} \\ W_{B} & \text { if } & r_{B} \geq r_{A}\end{array}\right.$

To illustrate the above, consider the following example.

Example 2.3.3: Suppose $A=\left\{\frac{1 \mathrm{e}^{\mathrm{i} \times 0}}{-1}, \frac{0.4 \mathrm{e}^{\mathrm{i} \pi}}{0}, \frac{0.8 \mathrm{e}^{\mathrm{i} \pi / 2}}{1}\right\}$ and $B=\left\{\frac{0.2 \mathrm{e}^{\mathrm{i} 3 \pi / 4}}{-1}, \frac{0.3 \mathrm{e}^{\mathrm{i} 2 \pi}}{0}, \frac{1 \mathrm{e}^{\mathrm{i} \pi / 5}}{1}\right\}$

Then $A \cup B=\left\{\frac{1 \mathrm{e}^{0 \pi}}{-1}, \frac{0.4 \mathrm{e}^{\mathrm{i} \pi}}{0}, \frac{1 \mathrm{e}^{\mathrm{i} \pi / 5}}{1}\right\}$

Here the union operation is (1) of definition 2.3.1.

\subsection{Fuzzy Complex Intersection}

It should be noted that the derivation of fuzzy complex intersection closely related to complex fuzzy union, which clear from the following definition.

\subsubsection{Definition([6,7])}

The intersection for two complex fuzzy set $\mathrm{A}$ and $\mathrm{B}$ on $\mathrm{U}$ defined as

$$
A \cap B=\left[r_{A}(x) * r_{B}(x)\right] e^{i w_{A \cap B}(x)}
$$

where $*$ is denoted as $\mathrm{T}-$ norm.

Example 2.4.1. From the example 2.3.3, we can find the fuzzy intersection of the two complex fuzzy sets A and B as follows.

$$
A \cap B=\left\{\frac{0.2 \mathrm{e}^{\mathrm{i} 3 \pi / 4}}{-1}, \frac{0.3 \mathrm{e}^{\mathrm{i} 2 \pi}}{0}, \frac{0.8 \mathrm{e}^{\mathrm{i} 5 \pi / 2}}{1}\right\}
$$

Note: The fuzzy union and intersection are not unique, As this depend on the type of functions used in T-Conorm and T-norms.

\subsection{Complex Fuzzy Complement}

We known that the complement of a fuzzy set A on $\mathrm{U}$ with membership function $\mu_{A}(x)$ is defined as, $\left(1-\mu_{A}(x)\right.$.)

The similar concept can be applied to the complex fuzzy set. But it is not always true for some cases. To illustrate this, consider the following example.

Example 2.5.1. Suppose the membership function of a complex fuzzy set is $\mu_{s}(x)=1 e^{i \pi}$. 
That is, $\mu_{s}(x)=1 e^{i \pi}=1[\cos \pi+i \sin \pi]=-1$

So the complement of $\mu_{s}(x)=1 e^{i \pi}$ is $\mu_{s}(x)=1-(-1)=2$, which is outside of the unit circle. This is violating of the closure properties of Fuzzy complex number.

2.5.1. Definition $([6,7])$. Suppose A is a fuzzy set defined as $U$. The complex fuzzy complement for the fuzzy set $\mathrm{A}$ is defined as $\left(1-\mu_{A}(x)\right)$,

where $\mu_{A}(x)=r_{s}(x)\left[\cos w_{s}(x)+\sin w_{s}(x)\right]$

This form of complex fuzzy complement does not reduce to its traditional fuzzy logic, if $w_{s}(x)=0$. That is,

when $\mu_{s}(x)=0, C\left(\mu_{s}(x)\right)=\left[1-r_{s}(x)\right]+i$ which is again a complex number.

Hence we can write $C\left(\mu_{s}(x)\right)=C\left(r_{s}(x)\right) \cdot e^{i c\left(w_{s} x\right)}$

Consider the function $\mathrm{C}:[0,1] \rightarrow[0,1]$, if $\mu_{s}(x)=C\left(\mu_{s}(x)\right)$ then the equation (13) holds. So we need $\left|C \cdot \mu_{s}(x)\right|=C\left(\left|\mu_{s}(x)\right|\right)$ to satisfy the equation (13). This can be achieved by adding $\pi$ to $w_{s}(x)$.

Hence the complement of $w_{s}(x)$ will be $w_{s}(x)+\pi$ that is $C\left(\mu_{s}(x)\right)=C \mu_{s}(x)+\pi$.

\subsection{Operation on Complex Fuzzy numbers [8]}

Let $\tilde{z}_{1}=\tilde{a}+i \tilde{b}$ and $\tilde{z}_{2}=\tilde{c}+i \tilde{d}$ be two complex fuzzy numbers where $\tilde{a}, \tilde{b}, \tilde{c}, \tilde{d}$ are fuzzy number with membership function $\mu_{\tilde{a}}(x), \mu_{\tilde{b}}(x), \mu_{\tilde{c}}(x), \mu_{\tilde{d}}(x)$

respectively. The basic arithmetic operation on $\tilde{z}_{1}$ and $\tilde{z}_{2}$ are defined as follows.

\subsubsection{Addition}

$\tilde{z}_{1}+\tilde{z}_{2}=(\tilde{a}+\tilde{c})+i(\tilde{b}+\tilde{d})$,

Where $\tilde{a}+\tilde{c}$ and $\tilde{b}+\tilde{d}$ are newly derived fuzzy numbers with the following membership functions:

$$
\begin{aligned}
& \mu_{\tilde{a}+\tilde{c}}(y)=\underset{y=x_{1}+x_{2}}{V}\left(\mu_{\tilde{a}}\left(x_{1}\right) \wedge \mu_{\tilde{c}}\left(x_{2}\right)\right) \\
& \mu_{\tilde{b}+\tilde{d}}(y)=\underset{y=x_{1}+x_{2}}{V}\left(\mu_{\tilde{b}}\left(x_{1}\right) \wedge \mu_{\tilde{d}}\left(x_{2}\right)\right) .
\end{aligned}
$$

\subsubsection{Subtraction}

$$
\tilde{z}_{1}-\tilde{z}_{2}=(\tilde{a}-\tilde{c})+i(\tilde{b}-\tilde{d})
$$

Where $\tilde{a}-\tilde{c}$ and $\tilde{b}-\tilde{d}$ are newly derived fuzzy numbers with the following membership functions : 


$$
\begin{aligned}
& \mu_{\tilde{a}-\tilde{c}}(y)=\underset{y=x_{1}-x_{2}}{V}\left(\mu_{\tilde{a}}\left(x_{1}\right) \wedge \mu_{\tilde{c}}\left(x_{2}\right)\right) \\
& \mu_{\tilde{b}-\tilde{d}}(y)=\underset{y=x_{1}-x_{2}}{V}\left(\mu_{\tilde{b}}\left(x_{1}\right) \wedge \mu_{\tilde{d}}\left(x_{2}\right)\right) .
\end{aligned}
$$

\subsubsection{Multiplication}

$$
\tilde{z}_{1} \times \tilde{z}_{2}=(\tilde{a} \tilde{c}-\tilde{b} \tilde{d})+i(\tilde{b} \tilde{c}+\tilde{a} \tilde{d})
$$

Where $\tilde{a} \tilde{c}-\tilde{b} \tilde{d}$ and $\tilde{b} \tilde{c}-\tilde{a} \tilde{d}$ are newly derived fuzzy numbers with the following membership functions.

$$
\begin{aligned}
& \mu_{\tilde{a} \tilde{c}-\tilde{b} \tilde{d}}(y)=\underset{y=x_{1} x_{2}-x_{3} x_{4}}{V}\left(\mu_{\tilde{a}}\left(x_{1}\right) \wedge \mu_{\tilde{c}}\left(x_{2}\right) \wedge \mu_{\tilde{b}}\left(x_{3}\right) \wedge \mu_{\tilde{d}}\left(x_{4}\right)\right) \\
& \mu_{\tilde{b} \tilde{c}-\tilde{a} \tilde{d}}(y)=\underset{y=x_{1} x_{2}+x_{3} x_{4}}{V}\left(\mu_{\tilde{b}}\left(x_{1}\right) \wedge \mu_{\tilde{c}}\left(x_{2}\right) \wedge \mu_{\tilde{a}}\left(x_{3}\right) \wedge \mu_{\tilde{d}}\left(x_{4}\right)\right)
\end{aligned}
$$

\subsubsection{Division}

$$
\frac{\tilde{z}_{1}}{\tilde{z}_{2}}=\left(\frac{\tilde{a} \tilde{c}+\tilde{b} \tilde{d}}{\tilde{c}^{2}+\tilde{d}^{2}}\right)+i\left(\frac{\tilde{b} \tilde{c}-\tilde{a} \tilde{d}}{\tilde{c}^{2}+\tilde{d}^{2}}\right)
$$

For notational simplicity, let $\tilde{t}_{1}=\frac{\tilde{a} \tilde{c}+\tilde{b} \tilde{d}}{\tilde{c}^{2}+\tilde{d}^{2}}$ and $\tilde{t}_{2}=\frac{\tilde{b} \tilde{c}+\tilde{a} \tilde{d}}{\tilde{c}^{2}+\tilde{d}^{2}}$ where $\tilde{t}_{1}$ and $\tilde{t}_{2}$ are newly derived fuzzy numbers with the following membership functions:

$$
\begin{aligned}
& \mu_{\tilde{t}_{1}}(y)=\underset{y=\frac{x_{1} x_{3}+x_{2} x_{4}}{x \frac{2}{3}+x \frac{2}{4}}, x \frac{2}{3}+x \frac{2}{4} \neq 0}{V}\left(\mu_{\tilde{a}}\left(x_{1}\right) \wedge \mu_{\tilde{b}}\left(x_{2}\right) \wedge \mu_{\tilde{c}}\left(x_{3}\right) \wedge \mu_{\tilde{d}}\left(x_{4}\right)\right) \\
& \mu_{\tilde{t}_{2}}(y)=\underset{y=\frac{x_{2} x_{3}-x_{1} x_{4}}{x \frac{2}{3}+x \frac{2}{4}}, x \frac{2}{3}+x \frac{2}{4} \neq 0}{V}\left(\mu_{\tilde{a}}\left(x_{1}\right) \wedge \mu_{\tilde{b}}\left(x_{2}\right) \wedge \mu_{\tilde{c}}\left(x_{3}\right) \wedge \mu_{\tilde{d}}\left(x_{4}\right)\right)
\end{aligned}
$$

\subsubsection{Modulus}

Given $\tilde{z}_{1}=\tilde{a}+i \tilde{b}$, the modulus of $\tilde{z}_{1}$ is defined:

$$
\left|\tilde{z}_{1}\right|=\sqrt{\tilde{a}^{2}+\tilde{b}^{2}}
$$

It is obvious that $\left|\tilde{z}_{1}\right|$ is a newly derived fuzzy number with the following membership function:

$$
\mu_{\left|\tilde{z}_{1}\right|}(y)=\underset{y=\sqrt{\frac{x^{2}}{1}+\frac{x^{2}}{2}}}{V}\left(\mu_{\tilde{a}}\left(x_{1}\right) \wedge \mu_{\tilde{b}}\left(x_{2}\right)\right)
$$




\subsection{COMPLEX FuZzY RELATION}

In this section, the complex fuzzy relation is introduced. Here discussion is limited to the relation between two complex fuzzy set.

2.7.1. Definition (Complex Fuzzy Relation) ([10]): Let A and B be two complex fuzzy sets defined on $\mathrm{U}$. Then

$$
\mu_{A}(x)=r_{A}(x) \cdot e^{i w_{A}(x)} \text { and } \mu_{B}(x)=r_{B}(x) \cdot e^{i w_{B}(x)}
$$

are their corresponding membership values. We can say $A \leq B$ if and only if both amplitude and phase of $\mathrm{A}$ and $\mathrm{B}$ are $A \leq B$ that is $r_{A}(x) \leq r_{B}(x)$ and $w_{A}(x) \leq w_{B}(x)$.

A complex fuzzy relation $\mathrm{R}(\mathrm{U}, \mathrm{V})$ is a complex fuzzy subset of the product space $\mathrm{U} x \mathrm{~V}$. The relation $\mathrm{R}(\mathrm{U}, \mathrm{V})$ is characterized by a complex membership function

$$
\begin{aligned}
& \mu_{R}(x, y), x \in U, y \in V \\
& \text { Where } R(U, V)=\left\{\left((x, y), \mu_{R}(x, y)\right) \mid \forall(x, y) \in U \times V\right\}
\end{aligned}
$$

Here the membership function $\mu_{R}(x, y)$ has the value within an unit circle in the complex plane with the from $\mu_{R}(x, y)=r(x, y) \cdot e^{i w(x, y)}$

where $e^{i w(x, y)}$ is a periodic function defined as $w(x, y)=w(x, y)+2 k \pi, k=0, \pm 1, \pm 2$

\subsubsection{Definition ([10])}

Let $\mathrm{X}, \mathrm{Y}$ and $\mathrm{Z}$ be three different Universes. Suppose A be a complex fuzzy relation defined from $\mathrm{X}$ to $\mathrm{Y}$ and $\mathrm{B}$ be a complex fuzzy relation defined from $\mathrm{Y}$ to $\mathrm{Z}$. Then composition of $\mathrm{A}$ and $\mathrm{B}$ denoted on $\mathrm{AoB}$ is a complex fuzzy relation defined from $\mathrm{X}$ to $\mathrm{Z}$ which can be represented as

$\mu_{A o B}(x, z)=r_{A o B}(x, z) \cdot e^{i w_{A o B}(x, z)}=\sup _{\forall y} \inf \left(r_{A}(x, y), r_{B}(y, z)\right) \cdot e^{i \sup \left(\inf \left(w_{A}(x, y), w_{B}(y, z)\right)\right.}$

\subsubsection{Definition (Complex Fuzzy Union Relation) ([10])}

Let $\mathrm{A}$ and $\mathrm{B}$ be two complex fuzzy relations defined on $\mathrm{U} \times \mathrm{V}$. The corresponding membership functions of $\mathrm{A}$ and $\mathrm{B}$ are defined as

$$
\mu_{A}(x, y)=r_{A}(x, y) \cdot e^{i w_{A}(x, y)} \text { and } \mu_{B}(x, y)=r_{B}(x, y) \cdot e^{i w_{B}(x, y)} \text { respectively. }
$$

The complex fuzzy union relation of $\mathrm{A}$ and $\mathrm{B}$ is defined as $\mathrm{A} \cup \mathrm{B}$ by membership function $\mu_{A \cup B}(x, y)$ where

$\mu_{A \cup B}(x, y)=r_{A U B}(x, y) \cdot e^{i w_{A U B}(x, y)}=\max \left(r_{A}(x, y), r_{B}(x, y)\right) \cdot e^{i \max \left(w_{A}(x, y), w_{B}(x, y)\right)}$

where $r_{A \cup B}(x, y)$ is a relation valued function on $[0,1]$ and $e^{i w_{A \cup B}(x, y)}$ is a periodic function defined in (18) 


\subsubsection{Definition (Complex Fuzzy Intersection Relation) ([10])}

Let $\mathrm{A}$ and $\mathrm{B}$ be two complex fuzzy sets defined on $\mathrm{U} x \mathrm{~V}$ with their corresponding membership functions as

$$
\mu_{A}(x, y)=r_{A}(x, y) e^{i w_{A}(x, y)} \text { and } \mu_{B}(x, y)=r_{B}(x, y), e^{i w_{B}(x, y)}
$$

respectively. The complex fuzzy intersection relation of $\mathrm{A}$ and $\mathrm{B}$ is defined as $\mathrm{A} \cap \mathrm{B}$ by membership function $\mu_{A \cap B}(x, y)$

$$
\begin{aligned}
\text { where } \mu_{A \cap B}(x, y)=r_{A \cap B}(x, y) \cdot e^{i w_{A \cap B}(x, y)} \\
=\min \left(r_{A}(x, y), r_{B}(x, y)\right) \cdot e^{i \min \left(w_{A}(x, y), w_{B}(x, y)\right)}
\end{aligned}
$$

Here $r_{A \cap B}(x, y)$ is a relation valued function on $[0,1]$ and $e^{i w_{A \cap B}(x, y)}$ is a periodic function.

\section{3. $\Delta$-EQUALITIES OF COMPLEX FUZZY RELATION [8]}

This section introduces $\delta$-equalities of complex fuzzy relation and discusses associated theories. Also introduces implication operators of fuzzy rules and exams complex fuzzy inference in the framework of $\delta$-equalities of complex fuzzy relations.

Definition 3.1 A distance of complex fuzzy sets is a function

$\rho:\left(F^{*}(U \times V), F^{*}(U \times V)\right) \rightarrow[0,1]$ with the properties: for any A, B, $\mathrm{C} \in \mathrm{F}^{*}(\mathrm{U} \times \mathrm{V})$

(1) $\rho(A, B) \geq 0, \rho(A, B)=0$ If and only if $\mathrm{A}=\mathrm{B}$,

(2) $\rho(A, B)=\rho(B, A)$,

(3) $\rho(A, B) \leq \rho(A, C)+\rho(C, B)$.

In the following, we introduce a function $\mathrm{d}$, which plays a key role in the rest of paper. We define $d(A, B)=\max \left(\sup _{(x, y) \in U \times V}\left|r_{A}(x, y)-r_{B}(x, y)\right|\right)$,

$$
\left.\frac{1}{2 \pi} \sup _{(x, y) \in U \times V}\left|\omega_{A}(x, y)-\omega_{B}(x, y)\right|\right)
$$

Obviously, this function d (., .) is closure for any relations between two complex fuzzy sets defined is Section 2, for example, Complex fuzzy union and complex fuzzy intersection, etc.

Example 3.1 Let

$$
\begin{gathered}
A=\frac{0.6 e^{i 1.2 \pi}}{-1}+\frac{1.0 e^{i 2 \pi}}{0}+\frac{0.8 e^{i 1.6 \pi}}{1}+\frac{0.5 e^{i \pi}}{2} \\
A^{\prime}=\frac{0.7 e^{i 1.4 \pi}}{-1}+\frac{1.0 e^{i 2 \pi}}{0}+\frac{0.6 e^{i 1.2 \pi}}{1}+\frac{0.4 e^{i 0.8 \pi}}{2} \\
\text { We see } \sup _{(x, y) \in U \times V}\left|r_{A}(x, y)-r_{A^{\prime}}(x, y)\right|=0.2 \quad \text { and } \\
\frac{1}{2 \pi} \sup _{(x, y) \in U \times V}\left|\omega_{A}(x, y)-\omega_{A^{\prime}}(x, y)\right|=0.2 . \text { Therefore } \\
\mathrm{d}\left(\mathrm{A}, \mathrm{A}^{\prime}\right)=0.2
\end{gathered}
$$

It is easy to see that, if $A$ and $B$ are two real fuzzy sets on $U \times V$, then 


$$
d(A, B) \sup _{(x, y) \in U \times V}\left|\mu_{A}(x, y)-\mu_{B}(x, y)\right|
$$

Definition 3.2 [ Cai 1995] Let $\mathrm{U}$ be a universe of discourse. Let A and B be two fuzzy sets on $\mathrm{U}$, and $\mu_{A}(x)$ and $\mu_{B}(x)$ their membership functions, respectively. Then $\mathrm{A}$ and $\mathrm{B}$ are said to be $\delta$ equal, denoted by $\mathrm{A}=(\delta) \mathrm{B}$, if any only if

$$
\sup _{x \in U}\left|\mu_{A}(x)-\mu_{B}(x)\right| \leq 1-\delta, \quad 0 \leq \delta \leq 1 .
$$

In this way, we say A and B construct an $\delta$-equality.

\section{Lemma 3.1 Let}

Then

$$
\delta_{1} * \delta_{2}=\max \left(0, \delta_{1}+\delta_{2}-1\right) ; 0 \leq \delta_{1}, \delta_{2} \leq 1 .
$$

(1) $0 * \delta_{1}=0 ; \forall \delta_{1} \in[0,1]$,

(2) $1 * \delta_{1}=\delta_{1} ; \forall \delta_{1} \in[0,1]$,

(3) $0 \leq \delta_{1} * \delta_{2} \leq 1 ; \forall \delta_{1}, \delta_{2} \in[0,1]$,

(4) $\delta_{1} \leq \delta_{11}^{\prime} \Rightarrow \delta_{1} * \delta_{2} \leq \delta_{11}^{\prime} * \delta_{2} ; \forall \delta_{1}, \delta_{1}^{\prime}, \delta_{2} \in[0,1]$,

(5) $\delta_{1} * \delta_{2}=\delta_{2} * \delta_{1} ; \forall \delta_{1}, \delta_{2} \in[0,1]$,

(6) $\left(\delta_{1} * \delta_{2}\right) * \delta_{3}=\delta_{1} *\left(\delta_{2} * \delta_{3}\right) ; \forall \delta_{1}, \delta_{2}, \delta_{3} \in[0,1]$.

Lemma 3.2 Let $\mathrm{f}, \mathrm{g}$ be bounded real valued functions on a set $\mathrm{U}$. Then

$$
\begin{gathered}
\left|\sup _{x \in U} f(x)-\sup _{x \in U} g(x)\right| \leq \sup _{x \in U}|f(x)-g(x)| \\
\left|\inf _{x \in U} f(x)-\inf _{x \in U} g(x)\right| \leq \sup _{x \in U}|f(x)-g(x)|
\end{gathered}
$$

Definition 3.3 [Zhang et al. 2004) Let A and B be two complex fuzzy sets on U, and $\mu_{A}(x)=r_{A}(x) \cdot e^{i \omega_{A}(x)}$ and $\mu_{B}(x)=r_{B}(x) \cdot e^{i \omega_{B}(x)}$ their membership functions, respectively. Then $\mathrm{A}$ and $\mathrm{B}$ are said to be $\delta$-equal, denoted by $A=(\delta) B$, if and only if

$$
d(A, B) \leq 1-\delta ; 0 \leq \delta \leq 1 .
$$

Theorem 3.1 Let A, A' be complex fuzzy sets defined on $\mathrm{U}$, and B, B' be complex fuzzy sets defined on V. If $\mathrm{A}=\left(\delta_{1}\right) A^{\prime}$ and $\mathrm{B}=\left(\delta_{2}\right) B^{\prime}$, then $A \oplus B=\left(\min \left(\delta_{1}, \delta_{2}\right) A^{\prime} \oplus B^{\prime}\right.$.

Theorem 3.2 Let A, A' be complex fuzzy sets defined on $\mathrm{U}$, and $\mathrm{B}$, B' be complex fuzzy sets defined on $\mathrm{V}$. If $\mathrm{A}=\left(\delta_{1}\right) A^{\prime}$ and $\mathrm{B}=\left(\delta_{2}\right) B^{\prime}$, then $A \otimes B=\left(\min \left(\delta_{1}, \delta_{2}\right)\right) A^{\prime} \otimes B^{\prime}$.

Theorem 3.3 Suppose Dienes-Rescher implication operator is applied to the fuzzy rule 'If $\mathrm{X}$ is $\mathrm{A}$ then $\mathrm{Y}$ is $\mathrm{B}^{\prime}$, that is $I(A, B)=\bar{A} \oplus B$ or

$$
\mu_{1(A, B)}(x, y)=\max \left(1-r_{A}(x), r_{B}(y)\right) \cdot e^{i \max \left(2 \pi-\omega_{A}(x), \omega_{B}(y)\right)} . \text { Further, let } \mathrm{A}^{\prime} \text { and } \mathrm{B}^{\prime}
$$

be two complex fuzzy sets with $A=\left(\delta_{1}\right) A^{\prime}$ and $B=\left(\delta_{2}\right) B^{\prime}$, then 


$$
1(A, B)=\left(\min \left(\delta_{1}, \delta_{2}\right)\right) I\left(A^{\prime}, B^{\prime}\right)
$$

Theorem 3.4 Suppose Lukasiewicz implication operator is applied to the fuzzy rule 'If $\mathrm{X}$ is $\mathrm{A}$ then $\mathrm{Y}$ is $\mathrm{B}^{\prime}$, that is $I(A, B)=A * B$ or

$$
\mu_{1(A, B)}(x, y)=\left(r_{A}(x) * r_{B}(y)\right) \cdot e^{i\left(\omega_{A}(x) * \omega_{B}(y)\right)}
$$

Further, let $\mathrm{A}^{\prime}$ and B' be two complex fuzzy sets with $A=\left(\delta_{1}\right) A^{\prime}$ and $B=\left(\delta_{2}\right) B^{\prime}$, then

$$
1(A, B)=\left(\delta_{1} * \delta_{2}\right) I\left(A^{\prime}, B^{\prime}\right)
$$

Where

$$
\omega_{A}(x) * \omega_{B}(y)=2 \pi \max \left(0, \frac{\omega_{A}(x)}{2 \pi}+\frac{\omega_{B}(y)}{2 \pi}-1\right)
$$

Theorem 3.5 Suppose Zadeh implication operator is applied to the fuzzy rule 'If $\mathrm{X}$ is $\mathrm{A}$ then $\mathrm{Y}$ is $\mathrm{B}^{\prime}$, that is $I(A, B)=\bar{A} \oplus(A \otimes B)$ or

$$
\mu_{1(A, B)}(x, y)=\max \left(1-r_{A}(x), \min \left(r_{A}(x), r_{B}(y)\right)\right) \cdot e^{i \max \left(2 \pi-\omega_{A}(x), \min \left(\omega_{A}(x), \omega_{B}(y)\right)\right)}
$$

Further, let $\mathrm{A}^{\prime}$ and B' be two complex fuzzy sets with $A=\left(\delta_{1}\right) A^{\prime}$ and $B=\left(\delta_{2}\right) B^{\prime}$, then

$$
1(A, B)=\left(\min \left(\delta_{1}, \delta_{2}\right)\right) I\left(A^{\prime}, B^{\prime}\right)
$$

Theorem 3.6 Suppose Godel implication operator is applied to the fuzzy rule 'If $\mathrm{X}$ is $\mathrm{A}$ then $\mathrm{Y}$ is $\mathrm{B}^{\prime}$, that is $\mathrm{I}(\mathrm{A}, \mathrm{B})=\mathrm{A} \Theta \mathrm{B}$

$$
\begin{gathered}
\text { Or } \mu_{1(A, B)}(x, y)=r_{1(A, B)}(x, y) e^{i \omega_{1(A, B)}(x, y)}, \text { where } \\
r_{1(A, B)}(x, y)= \begin{cases}1 & \text { if } r_{A}(x) \leq r_{B}(y) \\
r_{B}(y) & \text { otherwise, }\end{cases} \\
\omega_{1(A, B)}(x, y)= \begin{cases}2 \pi & \text { if } \omega_{A}(x) \leq \omega_{B}(y) \\
\omega_{B}(y) & \text { otherwise }\end{cases}
\end{gathered}
$$

Further, let A' and B' be two complex fuzzy sets with $A=\left(\delta_{1}\right) A^{\prime}$ and $B=\left(\delta_{2}\right) B^{\prime}$, then

$$
I(A, B)=(\delta) I\left(A^{\prime}, B^{\prime}\right)
$$

Where $\delta=\max \left(\begin{array}{l}\max \left(\inf _{y} r_{B}(y), \inf _{y} r_{B^{\prime}}(y)\right) *\left(\inf _{y \in V} \min \left(r_{B}(y), r_{B}(y)\right),\right. \\ \frac{1}{2 \pi} \max \left(\inf _{y} \omega_{B}(y), \inf _{y} \omega_{B^{\prime}}(y)\right) *\left(\inf _{y \in V} \min \left(\omega_{B}(y), \omega_{B^{\prime}}(y)\right)\right.\end{array}\right)$

And

$$
\omega_{A}(x) * \omega_{B}(y)=2 \pi \max \left(0, \frac{\omega_{A}(x)}{2 \pi}+\frac{\omega_{B}(y)}{2 \pi}-1\right)
$$

From the above operation $\delta$ is not exactly related to $\mu_{A}(x), \mu_{A^{\prime}}(x)$ but is exactly related to $\mu_{B}(x), \mu_{B^{\prime}}(x)$.

Realistic interpretation in complex fuzzy set 


\section{PROBABILITY OF COMPLEX FUZZY SET.}

A lot of criticism of a fuzzy logic has come from the probability theory community. Due to the some confusion is caused by complicated relation ship between the CFL and probability. They are similar is certain respect, yet different in some other respect. Here we are reveal this mysterious relationship between CFL and probability. It is important that we understand their relationship clearly so that we can not clarity confusion such as CFL is a clever disguise of probability theory but also to learn use both technique appropriately such as benefit will maximized.

4.1. Definition: The probability of a complex fuzzy event A is a generalization of probability theory.

$\mathrm{P}(\mathrm{A})=\int \mu_{A}(x) \times p_{x}(x) d x$

If $X$ is continuous or

$\mathrm{P}(\mathrm{A})=\sum_{i} \mu_{A}\left(x_{i}\right) \times p_{x}\left(x_{i}\right) d x$

If $\mathrm{X}$ is discrete $\mathrm{P}_{\mathrm{X}}$ denotes the probability distribution function of $\mathrm{X}$. and $\mu_{A}\left(x_{i}\right)$ be characterized by the membership function of the form $z=r_{s} e^{i w_{s}(x)}$ on a unit circle.

More over let two event $\mathrm{A}$ and $\mathrm{B}$ of a sample Space .We know probability theory $\mathrm{P}(\mathrm{A} \cup \mathrm{B})=\mathrm{P}(\mathrm{A})+\mathrm{P}(\mathrm{B})-\mathrm{P}(\mathrm{A} \cap \mathrm{B})$. Does the Relationship hold for Complex Fuzzy event? Answer depends upon choice of complex fuzzy union.

\subsection{Definition (Conditional probability)}

Suppose A and B are two event of a Sample Space The conditional probability A given B defined as $\mathrm{P}(\mathrm{A} / \mathrm{B})=\frac{P(A \cap B)}{P(B)}$

\subsection{Definition}

Let A and B are two Complex Fuzzy Event of a Sample Space S. The Probability of both the Complex Fuzzy event defined by

$$
P(A \cap B)=\sum_{x \in S} \mu_{A}(x) \times \mu_{B}(x) \times p_{x}(x)
$$

The member ship function may be defined by (21) and (22).

Where $\mu_{A}(x) \times \mu_{B}(x)=(\tilde{a} \tilde{c}-\tilde{b} \tilde{d})+i(\tilde{b} \tilde{c}+\tilde{a} \tilde{d})$, if $\quad \mu_{A}(x)=a+i b$ and $\mu_{B}(x)=c+i d$.

As the probability is coming on the form of complex number we can evaluate modulo to get the final result defined by (19). 


\subsection{Definition}

If $\mathrm{A}$ and $\mathrm{B}$ are any two complex fuzzy event in a sample space $\mathrm{S}$ and $\mathrm{P}(\mathrm{A}) \neq 0$ The conditional probability A given $\mathrm{B}, \mathrm{P}(\mathrm{A} / \mathrm{B})=\frac{P(A \cap B)}{P(B)}$

Or

$P(A \cap B)=P(A / B) \times P(B)$

\subsection{Probabilistic Interpretation of CFS}

It is possible to give complex Fuzzy set a probabilistic interpretation viewing the membership degree of $\mathrm{x}$ in a complex fuzzy set $\mathrm{A}$ as a conditional probability of A given $\mathrm{x} . \mu_{A}(x)=P(A / x)$ Such probabilistic interpretation is consistent with a compliment operation of Complex Fuzzy set as

$$
\mu_{A^{c}}(x)=P\left(A^{c} / x\right)
$$

Which may be calculated by (16).

How ever the probabilistic interpretation does not help us in calculation the intersection or union of complex fuzzy set.

$$
\begin{aligned}
& \mu_{A \cap B}(x)=P(A \cap B / x) \\
& \mu_{A \cup B}(x)=P(A \cup B / x)
\end{aligned}
$$

From the probability theory

$$
\begin{aligned}
& P(A \cup B / x)=P(A / x)+P(B / x)-P(A \cup B / x) \\
& \mu_{A \cup B}(x)=P(A \cup B / x)
\end{aligned}
$$

Here we only established constraints between union and intersection. If A and B conditionally independent of $\mathrm{x}$ we get

$P(A \cup B / x)=P(A / x) \times P(B / x)$

If one is Subset of others

$$
P(A \cap B / x)= \begin{cases}P(A / x) & \text { if } A \subseteq B \\ P(B / x) & \text { if } B \subset A\end{cases}
$$

Or

$$
P(A \cap B / x)=\mathrm{m} \text { in }((P(A / x), P(B / x))
$$

\section{Theorem 4.1}

If $\mathrm{A}_{1} \mathrm{~A}_{2 \ldots} \ldots \ldots \quad \mathrm{A}_{\mathrm{k} .}$ are complex Fuzzy event of $\mathrm{U}$ such that $\sum_{i=1}^{n} \mu_{A_{i}}(x)=1 \forall x \in U$

$\mathrm{P}\left(\mathrm{A}_{\mathrm{i}}\right) \neq 0 \quad \mathrm{i}=1,2, . ., \mathrm{k}$ and $\mathrm{B}$ is the complex Fuzzy event of $\mathrm{U}, \mathrm{P}(\mathrm{B}) \neq 0$ 


$$
P\left(A_{j} / B\right)=\frac{P\left(B / A_{j}\right) \times P\left(A_{j}\right)}{\sum_{i=1}^{k} P\left(B / A_{i}\right) \times P\left(A_{i}\right)}
$$

\section{Proof:}

$\mathrm{P}(\mathrm{A} / \mathrm{B})=\frac{P(A \cap B)}{P(B)}$ By Defininition(4.4)

In order to show the right hand side of the equation we can expand the denominator of (46).

$$
\begin{aligned}
& \sum_{i=1}^{k} P\left(B / A_{i}\right) \times P\left(A_{i}\right) \\
= & \sum_{i=1}^{k} P\left(A_{i} \cap B\right) \\
= & \sum_{i=1}^{k} \sum_{x_{k}} \mu_{A_{i}}\left(x_{k}\right) \times \mu_{B}\left(x_{k}\right) \times P\left(x_{k}\right) \\
= & \sum_{x_{k}} \mu_{B}\left(x_{k}\right) \times P\left(x_{k}\right) \times\left(\sum \mu_{A_{i}}\left(x_{k}\right)\right) \\
= & \sum_{x_{k}} \mu_{B}\left(x_{k}\right) \times P\left(x_{k}\right) \\
= & P(\mathrm{~B})
\end{aligned}
$$

Since $P\left(\mathrm{~B} / \mathrm{A}_{\mathrm{j}}\right) \times P\left(\mathrm{~A}_{\mathrm{j}}\right)=P\left(\mathrm{~A}_{\mathrm{j}} \cap \mathrm{B}\right)$

Theorem is thus proved.

\section{CONCLuSiON}

The work presented in this paper is the novel frame work of complex fuzzy relation. In this paper the various properties and operation of complex fuzzy set as well as complex fuzzy relation are investigated. We also presented the complement of complex fuzzy relation as well probabilistic interpretation is presented. A further study is required to implement these notions in real life applications such as knowledge representation and information retrieval in a Complex plan corresponding of two variables which is not suitable for traditional fuzzy logic. Also it can be do the complex fuzzy measure to more general class as well as complex fuzzy inference rule.

\section{REFERENCE}

[1] Buckley J.J (1987) "Fuzzy Complex Number" in Proceedings of ISFK, Gaungzhou, China, pp.597700 .

[2] Buckley J.J (1989) "Fuzzy Complex Number", Fuzzy Sets \& Systems, vol33, pp 333-345.

[3] Buckley J.J (1991) "Fuzzy Complex Analysis I: Differentiation", Fuzzy Sets \&Systems, Vol 4l, pp. 269-284.

[4] Jang J.S.R, Sun C.T, Mizutani E, (2005) “Neuro-Fuzzy And Soft Computing”, PHI, New Delhi.

[5] Pedrycz and Gomide F, (2004) "Introduction To Fuzzy Set Analysis And Design", PHI, New Delhi. 
[6] Ramot Daniel, Menahem Friedman, Gideon Langholz and Abraham Kandel (2003) "Complex Fuzzy Logic", IEEE Transactions on Fuzzy Systems, Vol 11, No 4.

[7] Ramot Daniel, Menahem Friedman, Gideon Langholz and Abraham Kandel, (2002) "Complex Fuzzy Set", IEEE Transactions on Fuzzy Systems, Vol 10, No 2.

[8] Xin Fu, Qiang Shen, (2009) "A Novel Framework Of Complex Fuzzy Number And Its Application To Computational Modeling”, FUZZ -IEEE 2009, Korea, August 20-24.

[9] Zadeh L.A. (1965) "Fuzzy Sets", Information \& Control, Vol 8,pp 338-353.

[10] Zhang Guangquan, Tharam Singh Dillon, Kai-yung Cai Jun Ma and Jie Lu,(2010) " $\sigma$-Equality of Complex Fuzzy Relations", $24^{\text {th }}$ IEEE International Conference on Advance Information Networking and Application.

[11] G.Q Zhang (1992) "Fuzzy distance and limit of fuzzy number, fuzzy system and mathematics", vol 6,No 1 p21-28.

[12] G.Q Zhang (1992) "Fuzzy limit theory of fuzzy complex number, fuzzy Set system", vol 46,pp 227235.

\section{Authors}

Mr. Nilambar Sethi is working as an Associate Professor in the Department of Information Technology, Gandhi Institute of Engineering \& Technology (GIET), Gunupur, Orissa, India. He is under the supervision of Dr. S.K. Das and Dr. D. C. Panda for his Doctoral degree in the field of Complex fuzzy sets and its applications. $\mathrm{He}$ is a life member of ISTE. He has the research interests in the fields of Computer Security, Algorithm Analysis and Fuzzy Logic.

Dr. S. K. Das is working as a Reader (Associate Professor) and Head of the Department of Computer Science, Berhampur University, Berhampur, Orissa, India. He has more than 17 research publications in National and International Journals and conferences. He has supervised three Ph.D. scholars and more than 10 scholars are under supervision. He is a life member of IEEE, ISTE, SGAT, CSI and OITS. He has the research interests in the fields of Data Communication and Computer Networks, Computer Security, Internet and Web Technologies, Database Management Systems, Mobile Ad Hoc Networking and Applications.

Dr. D. C. Panda is working as an Assistant Professor in the Department of Electronics and Communication Engineering, Jagannath Institute of Technology and Management (JITM), Paralakhemundi, Orissa, India. He has more than 33 research publications in National and International Journals and conferences. He attended Several QIP of different Universities and Institutions of India. He is a reviewer of IEEE Antenna and Wave Propagation Letters and Magazine. He published a book "Electromagnetic Field Theory Simplified:- A New Approach for Beginners", which is under review. He is an exposure in Computational Electromagnetic Tools. He has the research interests in the fields of Soft Computing Techniques, Computational Electromagnetic, Microwave Engineering and MIMO Wireless Networks.
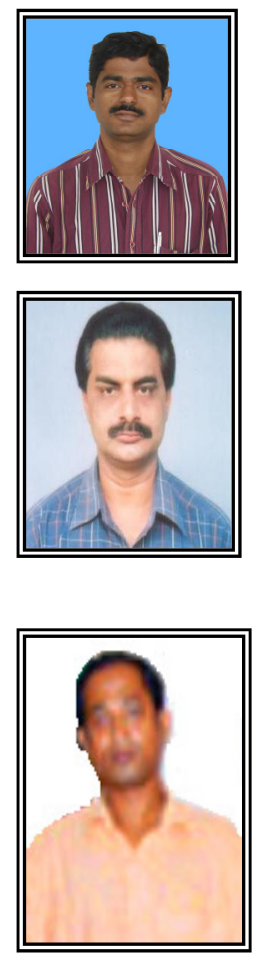